\title{
Einfluss des ökonomischen und sozialen Wandels auf die Bildungsteilhabe von Jugendlichen in Basel-Stadt, Freiburg, Luzern und Neuenburg 1950-1985
}

\author{
Miriam Baumeister, Pädagogische Hochschule Luzern und Universität Basel \\ Valérie Bürgy, Pädagogische Hochschule Luzern \\ Aurore Müller, Universität Freiburg
}

\begin{abstract}
Dieser Artikel behandelt die Bildungsteilhabe von 15-bis 19-jährigen Jugendlichen während der Bildungsexpansion. Die Autorinnen berücksichtigen die föderale Struktur des Bildungswesens, indem sie die vier Kantone Basel-Stadt, Freiburg, Luzern und Neuenburg vergleichen. Dabei gehen sie der Entwicklung der quantitativ-statistischen Verteilung der Jugendlichen auf die nachobligatorischen Bildungsangebote zwischen 1950 und 1980 nach und erklären diese anhand von ökonomischen, sozialen und bildungspolitischen Faktoren. Es kann gezeigt werden, dass die Bildungsexpansion eine höchst differenzierte Entwicklung war, die entscheidend von den kantonalen Gegebenheiten abhing.
\end{abstract}

\section{Einleitung}

In der Hochkonjunkturphase der Nachkriegsjahrzehnte rückte die Jugend in den Fokus der öffentlichen Diskurse (Bühler, 2016). Durch den fortschreitenden technologischen und wissenschaftlichen Fortschritt gewann in der Wirtschaft nach 1945 der sekundäre, aber auch der tertiäre Sektor zunehmend an Bedeutung. Dies machte es für die Arbeitnehmenden immer wichtiger, sich die für die Integration auf dem Arbeitsmarkt notwendigen Kenntnisse und Fähigkeiten anzueignen. Ab den 1950er-Jahren investierte die Schweizer Politik deswegen verstärkt in Forschung, Entwicklung und Ausbildung (Gugerli \& Tanner, 2017; Tanner, 2015). In der Folge stellten sich für junge Menschen in der Zeit nach dem Abschluss der obligatorischen Schulzeit die entscheidenden Weichen für den weiteren Lebensverlauf (Rieger, 2001). Die nachobligatorische Ausbildung wurde zur «Statuspassage» (Pätzold, 2008) in die Erwachsenenwelt.

Dieser Beitrag fokussiert auf die Jahre 1950 bis 1985. Er vergleicht und diskutiert anhand historischer statistischer Daten die Bildungsteilhabe in den Kantonen Basel-Stadt, Freiburg, Neuenburg und Luzern. Die Autorinnen gehen der Frage nach, wie sich die quantitative Verteilung der Jugendlichen auf die nachobligatorischen Bildungsangebote in den Vergleichskantonen im Untersuchungszeitraum entwickelt hat und welchen Einfluss die wirtschaftlichen, sozialen und politischen Unterschiede der Kantone darauf hatten. Diese Fragestellung ist eingebettet in das SNF-Projekt «Lebenswege fremdplatzierter Jugendlicher», an dem die Autorinnen beteiligt sind. ${ }^{1}$ Die vorliegende Fragestellung lotet somit die auf Basis der Statistik wahrscheinlichen Ausbildungswege für Jugendliche und deren kantonale Unterschiede aus.

Die Gesamtzahlen der Jugendlichen in Ausbildung werden mit den in den Volkszählungen erhobenen Beständen der 5-Jahresaltersklasse der 15-19-Jährigen in Beziehung gesetzt. Es wird davon ausgegangen, dass man grossmehrheitlich in dieser Altersgruppe einer nachobligatorischen Ausbildung nachging. Der Untersuchungszeitraum von 1950 bis 1985 deckt sich mit Bildungsreformen, die darauf abzielten, die Qualifikationen der Jugendlichen zu verbessern (Criblez, 2015). Die Auswahl der Vergleichskantone beruht auf dem bereits erwähnten SNF-Projekt und wurde einerseits kontrastiv und andererseits anhand der historischen Quellenlage getroffen. Sie stellt städtische und rurale Räume, verschiedene Konfessionen und unterschiedliche Sprachregionen und Wissenstraditionen gegenüber. Ziel des Beitrags ist es, die Entwicklung der Bildungsteilhabe der Jugendlichen vor dem Hintergrund der durch den Föderalismus bedingten wirtschaftlichen, sozialen und politischen Unterschiede in den Kantonen zu erklären.

1 Projekt der Pädagogischen Hochschule Luzern, Prof. Dr. Markus Furrer, und der Universität Freiburg, Prof. Dr. AnneFrançoise Praz, im Rahmen des Nationalfondsprogramm 76 «Fürsorge und Zwang» (verfügbar unter http://www.nfp76.ch/ de/projekte/massnahmen-und-lebenswege/projekt-furrer). Die Ergebnisse dienen in einer späteren Projektphase dazu, die Bildungsmöglichkeiten von Jugendlichen, die ausserfamiliär platziert wurden, zu vergleichen und vor dem jeweiligen sozioökonomischen Hintergrund einzuordnen. 
Es wird zunächst die methodische Vorgehensweise vorgestellt, gefolgt von der Darstellung der zentralen erhobenen Zahlen und ihres Vergleichs. Anschliessend werden die Ergebnisse in den jeweiligen kantonalen Kontext eingebettet, bevor ein allgemeines Resümee gezogen wird.

\section{Methode und Vorgehensweise}

Die Autorinnen bedienen sich der Methode des Vergleichs historischer statistischer Daten und der hermeneutischen Analyse zeitgenössischen Quellenmaterials sowie aktueller Sekundärliteratur, um eine gemeinsame Datenbasis zu erheben und gleichzeitig die Besonderheiten eines jeden Kantons zu berücksichtigen.

Die Hauptquellen sind die statistischen Jahrbücher des Kantons Basel-Stadt und die Staatsverwaltungsberichte der Kantone Freiburg, Neuenburg und Luzern. ${ }^{2}$ Daraus wurden systematisch vergleichbare statistische Daten erhoben. Die quantitativen Vergleichskriterien waren a) die Gesamtzahl der Jugendlichen von 15 bis 19 Jahren in nachobligatorischer Ausbildung, b) die Zahl der Jugendlichen, die eine höhere Schule besuchten und c) die Zahl derer, die eine Berufsausbildung absolvierten. ${ }^{3}$ Die Autorinnen mussten bei der Datenerhebung zu Gunsten der Vergleichbarkeit Lücken und Ungereimtheiten in Kauf nehmen. So konnte aufgrund der unzureichenden Datenlage leider keine Darstellung der Ergebnisse nach Geschlechtern oder sozialer Zugehörigkeit erfolgen. Dabei ist zu berücksichtigen, dass sich Schulstatistiken nicht losgelöst von politischen Prozessen betrachten lassen (Ruoss, 2018). Die Zusammenstellung der Daten und die Auswahl der bezifferten Bereiche hängen stark von den spezifischen kantonalen Bedürfnissen ab. Statistische Daten sind folglich nie neutral, sondern reflektieren vielmehr die jeweiligen Machtbeziehungen, in denen sie produziert wurden (Vuagniaux, 2011). Ausserdem hatte im Untersuchungszeitraum jeder Kanton eine eigene Herangehensweise, die Daten zu erheben und zu veröffentlichen, insbesondere vor 1970. Es ergibt sich folglich eine doppelte Konstruiertheit der Zahlen: einerseits bei der ursprünglichen Erhebung und andererseits bei der heutigen Aufarbeitung. Allerdings sind die Autorinnen überzeugt, dass aufgrund des bisherigen Mangels an vergleichenden Darstellungen die Vorteile der Erhebung die Nachteile überwiegen.

Diese statistische Datengrundlage wird daher auf der Basis von Sekundärliteratur reflektiert. Diese stellt die kantonalen Gegebenheiten ausreichend, wenn auch nicht immer umfassend, dar. Die zur Auswertung verwendeten Vergleichskriterien waren a) die ökonomische Struktur, b) die sozialpolitischen Faktoren und c) die Bildungspolitik. Ohne diese Informationen zur Entwicklung des obligatorischen und nachobligatorischen (Aus-)Bildungssystems sowie der wirtschaftlichen Entwicklung im jeweiligen kantonalen Kontext lassen sich die statistischen Aussagen nicht interpretieren.

\section{Forschungsstand zur Schweizer Bildungsgeschichte}

Mit dem vergleichenden Ansatz wollen die Autorinnen die Entwicklung der (Aus-)Bildung und der Bildungsteilhabe in der Schweiz aus einem neuen Blickwinkel betrachten. Schweizer Forschende haben sich in den vergangenen Jahren stark mit der historischen Bildungsforschung befasst. Vor zwanzig Jahren widmete sich eine Themennummer dieser Zeitschrift der Bildungsexpansion in der Schweiz mit Blick auf Systemdifferenzierung und -reformen, dabei wurden aber auch die Forschungsdesiderata insbesondere im quantitativen Bereich betont (Criblez \& Magnin, 2001). Relevante nachfolgende Beiträge zur Bildungsgeschichte behandelten den Bildungsraum Schweiz (Criblez, 2008) und diverse Schulreformen (Imlig, et al., 2018). Eine Reihe an Studien thematisiert die Geschichte der Berufsausbildung im überregionalen Vergleich (Bonoli, et. al., 2019; Gonon, 2002). In der Reihe Historische Bildungsforschung erscheinen 2022 zur Schweizer Bildungsgeschichte (Brühwiler et al., in Druck) und zu schulischen Wissensordnungen (Criblez et al., in Druck) zwei weitere Überblickswerke.

2 Zusätzlich dienten die Comptes-rendus annuels du Conseil d'État du canton de Fribourg, der Bericht des Regierungsrates des Kantons Luzern an den Grossen Rat über die geplante Staatsverwaltung, und die Rapports du Conseil d'État au Grand Conseil sur sa gestion et l'exécution des lois du canton de Neuchâtel als Quellen.

3 Dabei wurden die Kriterien b) und c) je Kanton noch deutlich detaillierter erhoben, so z. B. die Verteilung auf die verschiedenen Lehrberufe. 
Es wird deutlich, dass diese bisherige Forschung vor allem die übergeordnete eidgenössische Ebene oder einzelne Kantone untersucht. Die Bildungssoziologie betrachtet die Bildungsexpansion besonders im Hinblick auf die Entwicklung der Bildungschancen sowie ihre gewünschten und unerwünschten Auswirkungen (Hadjar \& Becker, 2006). Rolf Becker und Christoph Zangger (2013) konnten nachweisen, dass die Bildungsungleichheiten nach sozialer Herkunft und Geschlecht in der Schweiz zwischen 1970 und 1990 abnahmen, wobei sie 2016 auf die weiterhin «desolate Datengrundlage» hinwiesen. Andreas Rieger (2001) kommt in seiner detaillierten Analyse der Entwicklung des Mittelschulbesuchs im Kanton Zürich zum gleichen Ergebnis.

Es werden mit der Untersuchung der Sekundarstufe II nun zusätzlich die Entwicklung der Berufsbildung in den Fokus genommen. Zudem betrachten die Autorinnen die Entwicklung aus einem neuen Blickwinkel, indem sie die Auswirkungen der föderalen Bildungsorganisation und der kantonalen sozioökonomischen Gegebenheiten untersuchen. Die analysierten Unterschiede versprechen, die Frage nach unterschiedlichen Bildungschancen um den räumlichen Aspekt zu ergänzen, wie er für Deutschland bereits diskutiert wurde (Helbig \& Schneider, 2014; Peisert, 1967).

Im Folgenden werden die Ergebnisse der Studie in zwei Teilen ausgeführt. Zuerst werden die Ergebnisse des statistischen Vergleichs inklusive einiger allgemeiner Überlegungen dazu präsentiert. Anschliessend erfolgt die vergleichende Darstellung der ökonomischen, sozialen und bildungspolitischen kantonalen Kontextbedingungen.

\section{Bildungsteilhabe im Spiegel der Statistik}

Die zentralen Ergebnisse der Auswertung der historischen statistischen Daten sind in den untenstehenden Visualisierungen sowie in der Tabelle im Anhang (Abschnitt 9) ersichtlich. Die Daten wurden von den Autorinnen für den Zeitraum von 1950 bis 1985 jeweils in 5-Jahresschritten erhoben. Die Darstellungen bilden die Entwicklung der Anteile der Jugendlichen in Ausbildung an der Gesamtzahl von 15-19-Jährigen einmal in absoluten Zahlen und einmal prozentual ab. Sie basieren auf indikativen Zahlen, die den allgemeinen Trend der Entwicklung der postprimären Bildung in den vier Kantonen zeigen. Aufgrund der erwähnten Spezifika des historischen Vergleichs, erhebt die Darstellung nicht den Anspruch, einen genauen quantitativen Vergleich zu liefern. Die Kategorie «höhere Schulen» subsummiert Gymnasien, Handelsschulen und Lehrerseminare, während sich Berufslehren, landwirtschaftliche Ausbildungen und berufliche Vorkurse in der Kategorie «Berufsbildung» wiederfinden. Unter «Andere» finden sich Jugendliche, die entweder noch die obligatorische Schule besuchten, ein Zwischenjahr einlegten, einer ungelernten Arbeit nachgingen oder aber ihre Berufslehre bereits abgeschlossen hatten. Eine Aufschlüsselung dieser Gruppen wäre wertvoll gewesen, liess sich aus dem historischen Quellenmaterial allerdings leider nicht realisieren. Die Analyse der Sekundärliteratur in den Abschnitten 5, 6 und 7 legt nahe, dass aufgrund der Ausweitung der Schulpflicht mit einer Zunahme der Jugendlichen in der obligatorischen Schule in allen Kantonen zu rechnen ist. Ungelernte Tätigkeiten spielten vor allem in der Landwirtschaft sowie in den Westschweizer Industriebetrieben eine Rolle.

\section{Abbildung 1}

Visualisierung Ausbildungssituation in den Vergleichskantonen 1950-1985 in absoluten Zablen.

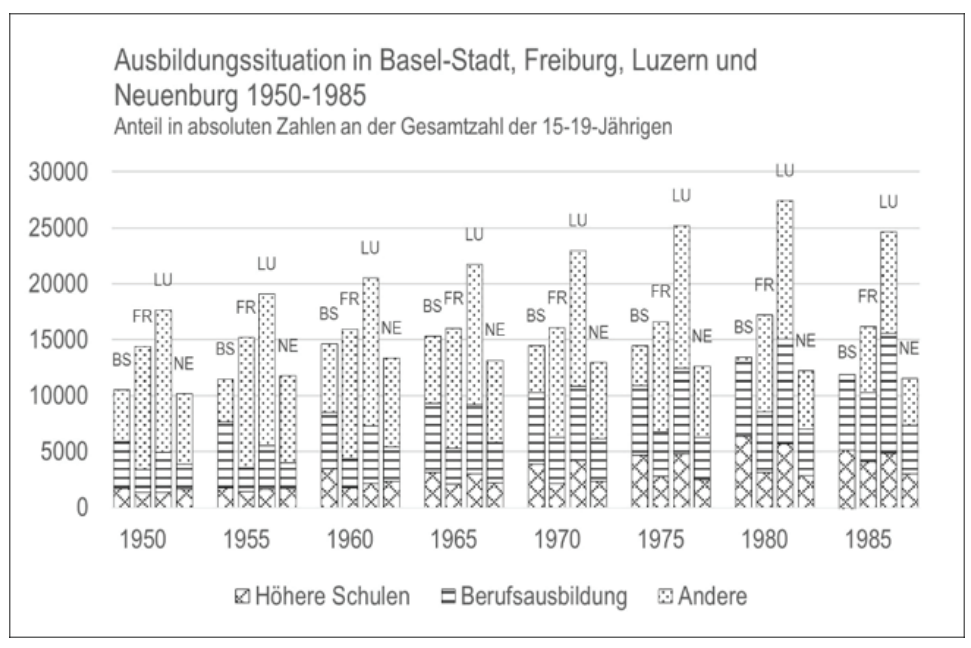

2021 SZBW 43 (3), DOI $10.24452 / \mathrm{sjer.43.3.3}$ 


\section{Abbildung 2}

Visualisierung Ausbildungssituation in den Vergleichskantonen 1950-1985 in Prozent.

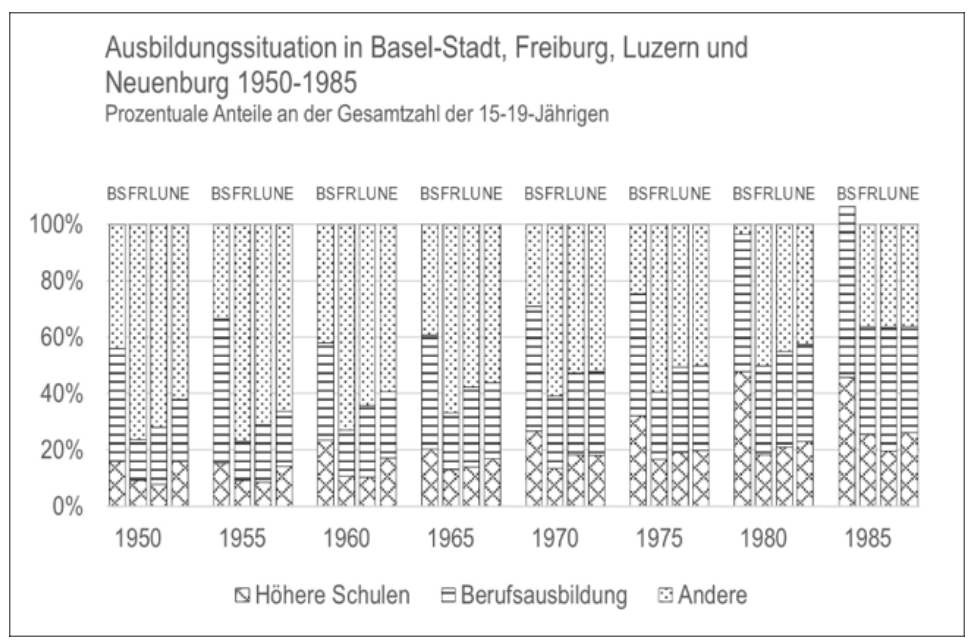

Die Auswertung der Verteilung der Jugendlichen auf die zwei unterschiedlichen Kategorien der Bildungsgänge zeigt, dass es in allen untersuchten Kantonen bis 1985 zu einer stetigen Zunahme der Bildungsteilhabe kam. Dabei weist die berufliche Ausbildung in den vier Kantonen durchwegs höhere Zahlen auf als die höhere Schulbildung. Der Kanton Basel-Stadt verzeichnete über den gesamten Untersuchungszeitraum die höchsten prozentualen Anteile: 1985 befanden sich im Vergleich zu 1950 doppelt so viele Jugendliche in den kantonalen Ausbildungsstätten. Dass die Ausbildungszahlen sogar die Gesamtzahl der Jugendlichen überschritten, ist auf eine im zentralen Stadtkanton hohe Zahl an Pendlerinnen und Pendlern aus der Agglomeration zurückzuführen.

Die Gesamtzahl der Ausbildungsplätze in den drei Kantonen Freiburg, Luzern und Neuenburg befindet sich prozentual zur wohnhaften Bevölkerung der Jugendlichen betrachtet auf einem tieferen, untereinander aber vergleichbaren Niveau. Insbesondere Luzern und Neuenburg weisen eine weitestgehend parallele prozentuale Entwicklung der Bildungsteilhabe auf, während Freiburg den vergleichbaren Schritt jeweils etwa fünf Jahre später vollzog.

In den Kantonen Basel-Stadt und Luzern absolvierten $1950 \mathrm{mehr}$ als doppelt so viele Jugendliche eine Berufsausbildung als eine höhere Schulausbildung. Bis 1980 hatte sich in Basel das Verhältnis zwischen höherer Schulbildung und beruflicher Ausbildung ausgeglichen. 1985 jedoch steigt der Anteil derer in einer beruflichen Ausbildung erneut an. 1950 gab es für 16\% der baselstädtischen Jugend einen Platz in einer höheren Schule, 1980 schon 48\% und im Jahre 1985 bereits 60\%. In Luzern hingegen stand im gesamten Untersuchungszeitraum die berufliche Ausbildung im Vordergrund. So ist an den Berufsschulen eine expansive Entwicklung der Schülerzahlen auszumachen: 1950 gab es für rund einen Fünftel der Jugendlichen in Luzern einen Lehrvertrag, 1980 bereits für einen Drittel. Doch die höhere Schulbildung verzeichnete auch in Luzern eine positive Entwicklung, aber auf tieferem Niveau.

Auch in den beiden Westschweizer Kantonen wird über den gesamten Zeitraum die berufliche Ausbildung prozentual häufiger gewählt als eine höhere Schulbildung. 1950 besuchte relativ zur Wohnbevölkerung der Jugendlichen im Kanton Freiburg nur einer von zehn Jugendlichen eine höhere Schule. Diese Zahl stieg bis 1985 auf ein Viertel an. Ähnlich verhielt es sich im Kanton Neuenburg. Hier stieg die Zahl von 16\% im Jahr 1950 auf 38\% im Jahr 1985 an. Bei der beruflichen Ausbildung markiert der Kanton Freiburg 1950 erneut das Schlusslicht mit 14\% im Jahr 1950. Zwischen 1960 und 1970 konnte dieser Anteil dort um 10\% auf rund ein Viertel erhöht werden, bis 1985 der Rückstand ausgeglichen und mit Neuenburg etwa gleich aufgeschlossen werden konnte. Auch in Freiburg befanden sich, ähnlich wie in Luzern und Neuenburg, 1985 ungefähr zwei Drittel der Jugendlichen in einem nachobligatorischen Bildungsangebot.

Die erhobenen Daten machen auch deutlich, dass die untersuchten Kantone ihr Bildungsangebot im Untersuchungszeitraum erweitert haben. Nicht nur die Gesamtzahl der Jugendlichen stieg, auch ihr Anteil in Ausbildung hat sich im Untersuchungszeitraum etwa verdoppelt. Dieser Anstieg ist einerseits auf eine neue Bundespolitik und andererseits auf wirtschaftliche Zwänge innerhalb der einzelnen Kantone zurückzuführen. Die Schweiz erlebte in der Nachkriegszeit bis in die 1970er-Jahre eine Phase des ausserordentlichen wirtschaftlichen Wachstums. Die ausserordentlich gute Konjunkturlage vermochte bis Mitte der 1970er-Jahre auch die 
geburtenstarken 1940er-Jahrgänge problemlos auf dem Arbeitsmarkt zu integrieren und ermöglichte Vollbeschäftigung (Lorenzetti, 2012). Damit einher ging der Ausbau der Sozialpolitik und des Bildungswesens.

Der Bund war in der Pflicht, ein höheres Bildungsniveau sicherzustellen, um wettbewerbsfähig zu bleiben und die Demokratisierung des Schulsystems zu fördern (Magnin \& Muller, 2012). Diese Entwicklung führte vor allem zu einem Ausbau des Mittelschulwesen, was eine direkte Folge auf die Hochschulen hatte (Herren, 2008). Gleichzeitig wurde auch die Gleichstellung der Geschlechter zunehmend auf eidgenössischer Ebene thematisiert (Mantovani Vögeli, 1994).

Die gesteigerte Wertschätzung der höheren Bildungsgänge führte gesamtschweizerisch bereits zwischen 1950 und 1965 zu einer Zunahme der Maturanden und Maturandinnen sowie der Anzahl der Studierenden. Zudem wurde in den Jahren 1963 und 1978 das Bundesgesetz über die Berufsbildung (BBG) revidiert, um bestehende Strukturen in der Berufslehre auf die steigenden Zahlen der abgeschlossenen Lehrverträge und zusätzlichen beruflichen Bildungseinrichtungen anzupassen (Büchel, 2018).

Die allgemeine Entwicklung der Beteiligung an nachobligatorischer Bildung in den Untersuchungskantonen entsprach also dem eidgenössischen Trend. Der Anstieg der Bildungsbeteiligung, der Verweildauer im Schulsystem und der Anzahl der höheren Schulabschlüsse spiegelt die Bildungsexpansion wider (Hadjar \& Becker, 2006). Aufgrund des ausgeprägten Föderalismus verlief dieser Prozess allerdings je nach Kanton unterschiedlich. Daher gehen die Autorinnen als nächstes anhand der drei gewählten Vergleichskriterien genauer auf die Spezifika der einzelnen Kantone ein, ohne Verständnis derer die statistischen Daten im historischen Vergleich nicht interpretiert werden können.

\section{Die wirtschaftliche Entwicklung der Vergleichskantone}

Vergleicht man die ökonomische Struktur, zeigt sich, dass der Strukturwandel und die Boomphase der Nachkriegsjahrzehnte die vier untersuchten Kantone in verschiedener Ausprägung erfasste und massgeblich Einfluss auf die Berufsbildung ausübte.

Die Basler Wirtschaft erlebte in den 1950er- und 1960er-Jahren eine rasante Expansion. Der Wandel vom sekundären, produzierenden Sektor zum tertiären Dienstleistungssektor setzte bereits in der Zwischenkriegszeit ein. Mitte des 20. Jahrhunderts arbeitete die Hälfte der erwerbstätigen Bevölkerung im tertiären Sektor. Die Tertiarisierung der Wirtschaftsstruktur wurde durch Rationalisierungsprozesse und Produktivitätssteigerungen begünstigt (Mooser, 2000). Die Landwirtschaft spielte in Basel-Stadt im Gegensatz zu den anderen untersuchten Kantonen keine Rolle mehr. Dies erklärt den deutlichen Anstieg in der Bildungsbeteiligung bereits in den 1950er-Jahren. In den 1970er-Jahren wurden, infolge geringeren Zuzugs und sinkender Geburtenrate, immer mehr Arbeitsplätze durch Personen aus den Nachbarkantonen und dem Ausland besetzt (Kreis, 2000; Mooser, 2000). Der produzierende Teil der Industrie wurde im Verlauf der 1970er-Jahre zunehmend aus der Stadt in die Agglomeration verlagert. Bis 1985 sank der Anteil der im industriellen Sektor Beschäftigten auf 37,4\%, im Dienstleistungssektor wuchs er auf 62,5\%.

Das katholisch-konservativ geprägte Freiburg hingegen war Ende des Zweiten Weltkriegs ein weitgehend landwirtschaftlicher Kanton. Noch in den 1950er-Jahren waren 35,4\% der arbeitenden Bevölkerung im primären Sektor tätig, wobei dieser aber zunehmende Veränderungen und Rationalisierung zu bewältigen hatte. Gleichzeitig expandierte der sekundäre Sektor und holte dank den koordinierten Anstrengungen der Behörden auf. Diese Industrialisierung zog grosse Arbeitgeber an (z. B. Ciba 1961, Polytype und Portescap 1962 oder Sika-Norm 1966). Der Sekundärsektor beschäftigte im Jahr 1970 43,4\% der Erwerbstätigen in Freiburg und löste die Dominanz der Landwirtschaft in der Wirtschaftsstruktur des Kantons ab. Der bereits thematisierte Anstieg in der Berufsausbildung 1965-1970 lässt sich darauf zurückführen. Darüber hinaus expandierte in dieser Zeit auch der tertiäre Sektor, der ab 1980 48,7\% der arbeitenden Bevölkerung beschäftigte (Piérart, 2005). Der Anteil war gegenüber Basel aber immer noch geringer.

Im deutschsprachigen und katholisch geprägten Kanton Luzern setzte in der Nachkriegszeit wie in den restlichen Landesteilen eine wirtschaftliche Hochkonjunktur ein, die bis zur Ölkrise von 1973 anhielt. Sie ging ebenfalls mit einem wirtschaftsstrukturellen Wandel einher, dieser begann aber im Vergleich zur Gesamtschweiz etwas verzögert. Der Landwirtschaftssektor verkleinerte sich wie in Freiburg stetig, lag 1950 jedoch über dem schweizerischen Durchschnitt. Industrie und Gewerbe hingegen expandierten in Luzern bis 1960 leicht unter dem schweizerischen Schnitt, wurden dafür aber nach 1965 etwas später vom allgemeinen Abwärtstrend erfasst. Dies spiegelt sich auch im moderaten aber recht gleichmässigen Anstieg der Bildungsteilhabe wider. Zwischen 1954 und 1973 zählte der sekundäre Sektor in Luzern die meisten Beschäftigten (zwischen 41\% und 44\%). Ab 
den 1960er-Jahren setzte im gesamten Kanton Luzern ein verstärkter Aufschwung im tertiären Sektor ein, der seit 1973, also früher als in Freiburg, der grösste Sektor ist (Hürlimann, 2013).

In Neuenburg war die kantonale Wirtschaft während des gesamten Untersuchungszeitraums durch die starke Dominanz des sekundären Sektors gekennzeichnet, in dem von 1950 bis 1980 zwischen 50 und 60\% der arbeitenden Bevölkerung beschäftigt waren. Von besonderer Bedeutung war die Uhrenindustrie, in der 1975 44,6\% der industriell Beschäftigten des Kantons arbeiteten, während es schweizweit nur 6,4\% waren (Schoepfer, 1982). Die Neuenburger Wirtschaft basierte folglich auf einer Spitzenindustrie, die einen hohen Bedarf sowohl an qualifizierten als auch unqualifizierten Arbeitskräften hatte. Laut Francesco Garufo (2015) bestand in der Uhrenindustrie die Tendenz, dass ungelernte Positionen hauptsächlich an Ausländer vergeben, während Arbeitsplätze für Ingenieure und Techniker mit im Kanton selbst ausgebildeten Fachkräften besetzt wurden. Dieser wirtschaftliche Fachkräftebedarf spiegelt sich, ähnlich wie in Basel, im, im Vergleich zu Freiburg und Luzern, deutlich höheren Anteil der Jugendlichen in höheren Schulen zu Beginn des Untersuchungszeitraums.

\section{Soziale und politische Rahmenbedingungen in den Kantonen}

Die jeweilige wirtschaftliche Entwicklung der untersuchten Kantone wurde begleitet und begünstigt durch Veränderungen der sozialen und politischen Faktoren. Der allgemeine Wohlstand wuchs. Gleichzeitig veränderten sich politische Strukturen und Inhalte und der Schweizer Sozialstaat entwickelte sich entlang des föderalen Systems und wirkte sich auf die Bildungsexpansion aus.

In Basel-Stadt fällt überraschend die Kontinuität sozialer Ungleichheit auf. Um die daraus resultierenden Herausforderungen abzufedern, wurden bereits früh politische Impulse gesetzt. So errang Basel-Stadt in der ersten Hälfte des 20. Jahrhunderts im Bereich der Bildungs-, Arbeitsmarkt- und Gesundheitspolitik eine Vorreiterrolle in der Schweiz und entwickelte sich zur «Wohlfahrtsstadt» (Mooser, 2000, S. 227). Bemerkenswerterweise wurden die wichtigsten sozialpolitischen Massnahmen dieser Pionierzeit unter bürgerlicher Dominanz getroffen (Degen, 2011). Dies gilt auch für die wichtigsten Bildungsreformen (1880, 1929 und 1988), wobei sich die Sozialdemokratie im 20. Jahrhundert als feste Grösse in der Basler Politik etablieren konnte (Mooser, 2000; Statistisches Amt Basel-Stadt, 2016). Ab den 1950er-Jahren verbesserte sich graduell der Zugang sozioökonomisch schlechter gestellter und weiblicher Jugendlichen zu höherer Bildung (Stirnimann, 2000).

Auch im Kanton Freiburg gingen die wirtschaftlichen Veränderungen mit einer entscheidenden politischen Transformation einher. Die seit 1881 mehrheitsführende christliche konservative Partei beherrschte über lange Zeit die staatlichen Institutionen und den Bildungsbereich. Die in den 1950er-Jahren einsetzenden wirtschaftlichen Transformationen schwächten jedoch die Wählerbasis der konservativen Partei so sehr, dass die allgemeinen Wahlen von 1966 das Ende ihrer Mehrheit im Grossen Rat zugunsten der Sozialdemokraten und Freisinnigen bedeuteten (Dorand, 2017). Die Veränderungen der 1960er-Jahre verbesserten in Freiburg die Bildungschancen und Arbeitsmarktintegration junger Menschen der Arbeiter- und Mittelschichten (Piérart, 2005).

Die Gegensätze zwischen Kirche und liberalem Staat sowie Stadt und Land prägten die massgeblichen Entwicklungen im Kanton Luzern (Arnold, 2013). Ähnlich wie im Kanton Freiburg verloren die Geistlichen ihren Einfluss im Bildungswesen erst nach dem Zweiten Vatikanischen Konzil (1962-1965) und der daraus resultierenden Öffnung der Kirche. Es kam zu einer Liberalisierung der Inhalte und der Organisation des Bildungssystems. Dies zeigt sich auch an der in absoluten Zahlen starken Zunahme des Besuchs höherer Schulen zwischen 1965 und 1980. Bis in die 1950er-Jahre diktierten die sozioökonomische und geografische Herkunft sowie das Geschlecht die Bildungschancen der Jugendlichen. Der gesellschaftspolitische Umbruch Mitte der 1960er-Jahre mit den Schlagworten Chancengleichheit und Bildung für alle änderten sich in Luzern die Bildungsdebatten grundlegend (Fischer, 2013).

Im Kanton Neuenburg gelang es den Freisinnigen im ausgehenden 19. Jahrhundert ihre politischen Ideale tief im Bildungswesen zu verankern (Barrelet, 2002). Dieser starke Einfluss der Parti radical (FDP/PLR) auf die Bildungspolitik wurde ab den 1960er-Jahren durch den wachsenden Einfluss der Linken ergänzt. In der Folge herrschte im Kanton mit einem bis zur Wirtschaftskrise von 1974 anhaltenden Gleichgewicht zwischen liberalen und sozialdemokratischen Kräften relative politische Stabilität. Diese Ausgewogenheit begünstigte eine Politik, die den Zugang zur Bildung erleichterte (Praz, 2006) und sich auch insbesondere in der Bildungsteilhabe in höheren Schulen widerspiegelt. 


\section{Kantonale Unterschiede in der Bildungspolitik}

Diese wirtschaftlichen und sozialpolitischen Veränderungen beeinflussten unmittelbar das Bildungswesen. Die föderale Autonomie der Kantone in der Bildungspolitik bedingt die Differenz der Bildungssysteme, deren Besonderheiten in diesem Abschnitt vorgestellt werden ${ }^{4}$.

In Basel-Stadt wurde bereits Ende des 19. Jahrhunderts die achtjährige Schulpflicht, die Unentgeltlichkeit aller öffentlichen Schulen und die Kostenfreiheit der Lehrmittel eingeführt und der Einsatz von Geistlichen als Lehrer verboten. 1964 wurde der obligatorische Schulbesuch auf neun Jahre erhöht. Während diese Faktoren den Bildungszugang und somit die Chancengleichheit begünstigten, fand noch bis 1988 bereits nach der vierjährigen Primarschule eine Frühselektion auf verschiedene weiterführende Schultypen nach Leistungsniveau statt (Felder, 2019). Und erst ab Ende der 1950er-Jahre wurde auf Primarschulebene und in den 1960erJahren an den Gymnasien die Koedukation eingeführt. Gleichzeitig wurde die weiterführende Bildung ab den 1950er-Jahren gezielt gefördert und ausgebaut. Triebfeder war der massive «Mangel an Ausgebildeten aller Stufen» (Stirnimann, 2000, S. 391) auf dem baselstädtischen Arbeitsmarkt. Ungelernte Arbeitskräfte hingegen waren immer weniger gefragt. 1971 wurde für «leistungswillige Lehrlinge» (Felder, 2019, S. 230) die Berufsmittelschule eröffnet. 1967 etablierte Basel-Stadt ein Stipendienwesen (Stirnimann, 2000). Es zeigt sich, dass Basel-Stadt als Universitätsstandort den Hochschulzugang gezielt zu fördern versuchte, die hohe Quote an Jugendlichen in höheren Schulen unterstreicht diese Bemühungen.

Im Kanton Freiburg trat 1950 die Mehrheit der Jugendlichen direkt nach dem Verlassen der obligatorischen Schule ins Berufsleben ein (Piérart, 2005), nur 24\% absolvierten eine nachobligatorische Ausbildung. Die wirtschaftliche Neuordnung nach dem Zweiten Weltkrieg veranlasste die Jugendlichen dazu, sich für Tätigkeiten zu entscheiden, die keine vorherige Lehre erforderten (ebd.). Unqualifizierte Arbeiten in der Landwirtschaft oder Industrie, aber auch Einsätze bei Rodungen und in Torfmooren boten den jungen Arbeitenden attraktive Gehälter. Die hohe Zahl der ungelernten Arbeitskräfte war weitgehend auf ein unzureichendes Bildungssystem zurückzuführen. Nur 12 bis 15\% der Jugendlichen besuchten überhaupt eine Sekundarschule, gegenüber 30\% in der übrigen Schweiz (Gachet, 2016); gemäss der Berechnung der Autorinnen sogar nur 9\% ein Schulangebot im Bereich der Sekundarstufe II. Das Sekundarschulgesetz von 1951 sollte den Zugang erleichtern. Der Eintritt in die Sekundarschule sowie die damit verbundene Möglichkeit auf eine Hochschulbildung blieb aber von den finanziellen Möglichkeiten der Eltern abhängig. Dies galt insbesondere für die Mädchen. Abgesehen von der Einrichtung einer formellen Mädchenschule im Seebezirk waren die Mädchenschulen bis Mitte der 1960erJahre in privater Hand und wurden von Nonnen geleitet (Python, 2002).

Durch den erhöhten Fachkräftebedarf der Freiburger Industrie in den 1960er-Jahren entstand ein echter politischer Wille, junge Menschen nach der obligatorischen Schulzeit zu einer postprimären Ausbildung zu ermutigen. Die Idee der Demokratisierung der Bildung leitete eine langsame Entwicklung des Sekundarschulwesens ein, die bis 1986 andauerte (Gachet, 2016), wobei der stärkste Zuwachs zwischen 1970 und 1975 sowie 1980 und 1985 verzeichnet wurde. Gleichzeitig war das Stipendienwesen noch kaum entwickelt und die Aufnahmeprüfungen an den Gymnasien blieben in Kraft. Die kantonalen Behörden konzentrierten sich stattdessen auf die Entwicklung der Lehrlingsausbildung, die für die Wirtschaft von unmittelbarem Nutzen war. Unter dem Anstoss des neuen Bundesgesetzes über die Berufsbildung von 1963 ergriff der Kanton konkrete Massnahmen zur Förderung des dualen Systems der Berufsausbildung (Bays, Cottet, Philipona, Steinauer, 2016). Diese Bemühungen zeigen sich in einem anteiligen Anstieg der Berufsausbildung von rund 10\% während der 1960er-Jahre.

Zwischen 1950 und 1965 nahm auch der Kanton Luzern eine expansive Schulentwicklung vor, die durch das Erziehungsgesetz von 1953 mit einigen Neuerungen vorangetrieben wurde (Pfenninger, 1998). So hat sich die Zahl der Schülerinnen und Schüler an höheren Schulen in dieser Zeitspanne mehr als verdoppelt. Die steigenden Schüler- und Klassenzahlen, die Schaffung von Oberschulen, der 3. Sekundarklasse und die Bemühungen, den Mittelschulbesuch zugänglicher zu machen, prägten diese Zeit. Aber es mussten auch immer noch viele junge Frauen im Anschluss an die Schulzeit eine hauswirtschaftliche Fortbildungsschule besuchen (Häfliger, 2002). Die Institutionen der höheren Schulbildung wurden durch zahlreiche Reformen in den 1960er-Jahren liberalisiert, demokratisiert und schrittweise für Mädchen geöffnet (Fischer, 2013). In Koope-

4 Für einen detaillierten Vergleich der Schulsysteme fehlt der Platz. Vgl. dazu die Untersuchungen zur Systementwicklung von Brühwiler et al. (im Druck). 
ration mit den restlichen Zentralschweizer Kantonen wurde vor allem auf den Ausbau der dualen Berufsbildung gesetzt, ein Technikum gegründet und stetig ausgebaut sowie bereits Mitte der 70er-Jahre eine Berufsmittelschule eingeführt. Die Gründung einer Universität hingegen wurde zunächst an der Urne abgelehnt (Büchel, 2018). Der Anteil der Jugendlichen in Berufsbildung erhöhte sich zwischen 1970 und 1985 um rund 15\%.

Im Kanton Neuenburg erleichterten die politischen Verhältnisse sowie die auf die fortschrittliche Uhrenindustrie ausgerichtete Wirtschaft schon früh den Zugang zur Sekundarschulbildung und zur beruflichen Weiterbildung und ermöglichten so den Unternehmen, hochqualifizierte Arbeitskräfte direkt vor Ort anzuwerben. Bereits 1919 wurde die Sekundarschule kostenfrei und wurde von Jungen und Mädchen gleichermassen besucht. Diese formale Chancengleichheit führte wie in Basel zu einer vergleichsweise hohen Schulbesuchsquote. Dennoch wurde sie erst 1962 obligatorisch und erst 1975 besuchten alle jungen Neuenburgerinnen und Neuenburger sie tatsächlich. Obwohl die formale Gleichberechtigung in diesem Kanton rasch verwirklicht wurde (1960 waren 50,8\% der Neuenburger Schülerschaft weiblich), verliessen viele Mädchen die Schule nach der obligatorischen Schulzeit, um ohne Ausbildung in den Arbeitsmarkt einzutreten (Ebel, 1981). Im Bereich der nach-obligatorischen Bildung entwickelte der Kanton neben der dualen Lehre zahlreiche Berufsschulen zur vollzeitschulischen Ausbildung (1935 gab es 40 in der ganzen Schweiz, darunter sieben im Kanton Neuenburg). Vervollständigt wurde das Angebot durch die Gründung eines Technikums im Jahr 1932 und die Eröffnung einer Ingenieurschule im Jahr 1978. Die Uhrenindustrie beförderte auch die Schaffung von Kunstschulen, kaufmännischen Ausbildungen und Gymnasien (Société d'histoire et d'archéologie du canton de Neuchâtel, 1993). Das Neuenburger Bildungssystem basierte auf einem Vollzeitschulmodell nach französischem Vorbild, im Gegensatz zum im deutschsprachigen Raum geprägten dualen Ausbildungssystem, das in Kantonen wie Freiburg, Luzern oder Basel umgesetzt wurde (Tabin, 1989). Dies veränderte die anteilsmässige Verteilung auf die Ausbildungsgänge allerdings nicht.

\section{Fazit und Ausblick: Entwicklung in Richtung Chancengleichheit?}

Bildung vollzieht sich im Kontext von demografischen, ökonomischen, politischen, kulturellen und sozialen Faktoren und Entwicklungen. Daher muss auch der Ausbau der Ausbildungsangebote und des Zugangs dazu kurz die Entwicklung der Bildungsteilhabe - im Untersuchungszeitraum vor diesem Hintergrund beschrieben und analysiert werden. Dies erlaubt, die Bildungsexpansion in der Schweiz differenzierter zu betrachten.

Im quantitativen Vergleich konnte gezeigt werden, dass in allen vier untersuchten Kantonen die Zahl der Jugendlichen in den nachobligatorischen Bildungsangeboten von 1950 bis 1980 stieg. Neben der anteiligen Verdoppelung erhöhte sich auch die Gesamtzahl der 15- bis 19-Jährigen. Ausser dem beachtlichen Zuwachs, sowohl in den höheren Schulen als auch die Berufsausbildung, fielen die kantonalen Unterschiede in der Verteilung auf die Bildungsgänge auf. Zur Interpretation dieser Ergebnisse wurde Sekundärliteratur herangezogen. Es zeigte sich, dass alle Vergleichskantone auf einen wirtschaftsstrukturellen Wandel und eine höhere Nachfrage nach fachlicher Ausbildung zu reagieren hatten. Die Entwicklungen vollzogen sich jedoch unterschiedlich, da sich die wirtschaftlichen Bedürfnisse nicht einheitlich entwickelten, sozial und politische Besonderheiten bestanden - und weil der Föderalismus eine grosse Autonomie der Kantonsregierungen bei der Gestaltung, Finanzierung und Umsetzung der bildungspolitischen Massnahmen ermöglichte.

Jeder Kanton reagierte dabei auf seine spezifischen Bedürfnisse: Im Kanton Neuenburg war die Ausrichtung auf die Uhrenindustrie ausschlaggebend. Die Kantone Freiburg und Luzern benötigten aufgrund ihrer wirtschaftlichen Entwicklung zunächst Fachkräfte im industriell-gewerblichen Sektor und bauten ihre dualen Bildungsangebote dementsprechend aus. Basel-Stadt sah sich von allen Vergleichskantonen am stärksten in der Pflicht, sein Angebot an höheren Schulen auszubauen. Während also die Kantone Basel-Stadt und Neuenburg, angetrieben durch den wirtschaftlichen Bedarf an Facharbeitenden, in die Ausbildung der Jugendlichen investierten und die Angebote modernisierten, die Kantone Luzern und Freiburg, die konservativer waren und sich vor allem in ländlichen Regionen am traditionellen Wirtschaftsmodell orientierten, längere Zeit am Rand dieser Entwicklung blieben. Die Analyse der Entwicklungen in Basel-Stadt, Freiburg, Neuenburg und Luzern mit vergleichenden Bezügen zeigt, dass das, was in der Literatur oft verkürzt als Bildungsexpansion angeführt wird, sich konkret und lokal noch sehr unterschiedlich auswirkte.

Was lässt sich auf Grundlage der Ergebnisse über die Chancen der Jugendlichen auf eine nachobligatorische Ausbildung sagen? Seit den 1960er-Jahren stellt Chancengleichheit ein Ideal in der Bildung dar, das auch heute noch nicht als erreicht gilt (Becker \& Schoch, 2018; Criblez, 2001). Es konnte gezeigt werden, dass die kantonale

2021 SZBW 43 (3), DOI 10.24452/sjer.43.3.3

Miriam Baumeister, Valérie Bürgy und Aurore Müller 
Herkunft beziehungsweise der räumlich erreichbare Kanton an sich bereits die Bildungschancen beeinflusste. So war es im reformierten Kanton Basel-Stadt ungleich wahrscheinlicher, eine höhere Schule zu besuchen als im katholischen Kanton Luzern oder Freiburg. In Neuenburg verhinderte der Einfluss der Wirtschaft auf die Politik einen stärkeren Ausbau der höheren Schulen zu Gunsten der Berufslehren. Hinsichtlich der Geschlechterverteilung und der sozialen Herkunft musste auf Sekundärliteratur zurückgegriffen werden ${ }^{5}$. Insbesondere das Beispiel Basel-Stadt macht deutlich, dass gleiche Chancen auf dem Papier nicht automatisch die tatsächliche Chancengleichheit bedingen. In Kantonen wie Neuenburg, die Reformen wie die Unentgeltlichkeit der Sekundarschule erst deutlich später einführten, zeigten diese Massnahmen deutlich unmittelbarere Wirkung. In allen vier Kantonen wurde der Zugang zu den Sekundar-, Mittel- und Berufsschulen für die unteren Schichten sowie für Mädchen erleichtert. Trotzdem sahen sich die Kantone weiterhin mit der Herausforderung konfrontiert, soziale Ungleichheiten bedingt durch den sozio-ökonomischen Hintergrund und das Geschlecht der Jugendlichen zu schliessen. Folglich hingen die Chancen beim Zugang der Jugendlichen einer höheren Schulbildung, zu einer Berufsausbildung und zum Arbeitsmarkt neben der sozioökonomischen und geografischen Zugehörigkeit sowie dem Geschlecht von den kantonalen Gegebenheiten ab. Die Annäherung an das Ideal der Chancengleichheit erfolgte stets nur so weit, wie es wirtschaftlich und politisch opportun erschien.

Bildungsreformen wirken in der Schweiz über eine «longue durée» (Osterwalder, 2018). Die Forschung zu Bildungsteilhabe und Bildungschancen heute zeigt, dass sich die Bildungsexpansion ambivalent ausgewirkt hat (Hadjar \& Becker, 2006). So stellten Zangger und Becker (2013) fest, dass die sozialen Ungleichheiten beim Erwerb der Studienberechtigung in den 1990er-Jahren wieder zunahmen. Judith Arnold (2013) konnte für die Zentralschweizer Kantone die Pfadabhängigkeit des negativen Einflusses der katholisch-konservativen Prägung auf die höhere Schulbildung bis ins Jahr 2000 feststellen. Jüngere Forschung wiederum hat gezeigt, dass nicht die Religion an sich, sondern die damit verbundene soziale Lage Auswirkungen auf den Bildungserfolg hat (Helbig \& Schneider, 2014). Für weitere Untersuchungen wird es entscheidend sein, die Intersektionalität von Ungleichheitsfaktoren zu fokussieren und nicht mehr zeitgemässe Auswirkungen der Bildungsexpansion im heutigen Bildungssystem zu identifizieren.

5 Eine Aufarbeitung historischer Quellen hinsichtlich entsprechender Daten wäre für die Forschung wünschenswert, aber müsste aufgrund der schwierigen Datenlage in Kleinarbeit in den Kantons- und Gemeindearchiven erfolgen. 


\section{Anhang}

Abbildung 3

Datentabelle zur Ausbildungssituation in den Vergleichskantonen 1950-1985 in absoluten Zablen und in Prozent.

\begin{tabular}{|c|c|c|c|c|}
\hline \multicolumn{5}{|c|}{ Ausbildungssituation in Basel-Stadt, Freiburg, Luzern und Neuenburg } \\
\hline \multicolumn{5}{|c|}{ Angaben in absoluten Zahlen und in Prozent (Anteil an der Gesamtzahl der 15-19-Jährigen) } \\
\hline & \multirow{2}{*}{\begin{tabular}{|l|} 
Bevölkerung 15-19 \\
Jahre total
\end{tabular}} & \multicolumn{2}{|c|}{$\begin{array}{l}\text { Verteilung der Jugendlichen auf die } \\
\text { verschiedenen nachobligatorischen } \\
\text { Bildungsrichtungen }\end{array}$} & \multirow{2}{*}{$\begin{array}{l}\text { Gesamtzahl in einer } \\
\text { nachobligatorischen } \\
\text { Ausbildung }\end{array}$} \\
\hline & & Höhere Schulen & $\begin{array}{l}\begin{array}{l}\text { Berufsausbildung } \\
\text { (inkl. Vorkurse) }\end{array} \\
\end{array}$ & \\
\hline \multicolumn{5}{|c|}{ Basel-Stadt } \\
\hline 1950 & $10517(100 \%)$ & $1674(16 \%)$ & $4199(40 \%)$ & $5873(56 \%)$ \\
\hline 1955 & $11450(100 \%)$ & $1764(15 \%)$ & $5860(51 \%)$ & $7624(67 \%)$ \\
\hline 1960 & $14647(100 \%)$ & $3454(24 \%)$ & $5040(34 \%)$ & $8494(58 \%)$ \\
\hline 1965 & $15324(100 \%)$ & $3127(20 \%)$ & $6175(40 \%)$ & $9302(61 \%)$ \\
\hline 1970 & $14471(100 \%)$ & $3856(27 \%)$ & $6403(44 \%)$ & $10259(71 \%)$ \\
\hline 1975 & $14450(100 \%)$ & $4650(32 \%)$ & $6255(43 \%)$ & $10905(75 \%)$ \\
\hline 1980 & $13439(100 \%)$ & $6413(48 \%)$ & $6551(49 \%)$ & $12964(96 \%)$ \\
\hline 1985 & $11190(100 \%)$ & $5122(46 \%)$ & $6755(60 \%)$ & $11877(106 \%)$ \\
\hline \multicolumn{5}{|c|}{ Freiburg } \\
\hline 1950 & $14409(100 \%)$ & $1330(9 \%)$ & $2087(14 \%)$ & $3417(24 \%)$ \\
\hline 1955 & $15179(100 \%)$ & $1393(9 \%)$ & $2108(14 \%)$ & $3501(23 \%)$ \\
\hline 1960 & $15948(100 \%)$ & $1731(11 \%)$ & $2578(16 \%)$ & $4309(27 \%)$ \\
\hline 1965 & $15988(100 \%)$ & $2083(13 \%)$ & $3215(20 \%)$ & $5298(33 \%)$ \\
\hline 1970 & $16027(100 \%)$ & $2160(13 \%)$ & $4101(26 \%)$ & $6261(39 \%)$ \\
\hline 1975 & $16614(100 \%)$ & $2756(17 \%)$ & $3962(24 \%)$ & $6718(41 \%)$ \\
\hline 1980 & $17200(100 \%)$ & $3149(18 \%)$ & $5412(31 \%)$ & $8561(49 \%)$ \\
\hline 1985 & $16142(100 \%)$ & $4100(25 \%)$ & $6172(38 \%)$ & $10272(63 \%)$ \\
\hline \multicolumn{5}{|c|}{ Luzern } \\
\hline 1950 & $17638(100 \%)$ & $1365(8 \%)$ & $3594(20 \%)$ & $4959(28 \%)$ \\
\hline 1955 & $19068(100 \%)$ & $1626(9 \%)$ & $3881(20 \%)$ & $5507(29 \%)$ \\
\hline 1960 & $20498(100 \%)$ & $2127(10 \%)$ & $5202(25 \%)$ & $7329(35 \%)$ \\
\hline 1965 & $21729(100 \%)$ & $3020(14 \%)$ & $6166(28 \%)$ & $9186(42 \%)$ \\
\hline 1970 & $22959(100 \%)$ & $4209(18 \%)$ & $6647(29 \%)$ & $10856(47 \%)$ \\
\hline 1975 & $25185(100 \%)$ & $4798(19 \%)$ & $7652(30 \%)$ & 12441 (49\%) \\
\hline 1980 & $27410(100 \%)$ & $5722(21 \%)$ & $9340(34 \%)$ & $15062(55 \%)$ \\
\hline 1985 & $24622(100 \%)$ & $4824(20 \%)$ & $10799(44 \%)$ & $15623(64 \%)$ \\
\hline \multicolumn{5}{|c|}{ Neuenburg } \\
\hline 1950 & $10184(100 \%)$ & $1629(16 \%)$ & $2240(22 \%)$ & $3869(38 \%)$ \\
\hline 1955 & $11783(100 \%)$ & $1667(14 \%)$ & $2311(20 \%)$ & $3978(34 \%)$ \\
\hline 1960 & $13383(100 \%)$ & $2302(17 \%)$ & $3133(23 \%)$ & $5435(40 \%)$ \\
\hline 1965 & $13162(100 \%)$ & $2214(17 \%)$ & $3574(27 \%)$ & $5788(44 \%)$ \\
\hline 1970 & $12941(100 \%)$ & $2347(18 \%)$ & $3835(30 \%)$ & $6182(48 \%)$ \\
\hline 1975 & $12637(100 \%)$ & $2519(20 \%)$ & $3764(30 \%)$ & $6283(50 \%)$ \\
\hline 1980 & $12234(100 \%)$ & $2817(23 \%)$ & $4176(34 \%)$ & $6993(57 \%)$ \\
\hline 1985 & $11544(100 \%)$ & $3012(26 \%)$ & $4359(38 \%)$ & $7371(64 \%)$ \\
\hline
\end{tabular}




\section{Bibliografie}

\section{Quellen}

Conseil d'État de Fribourg (Hrsg.). Comptes-rendus de l'administration du Conseil d'État du canton de Fribourg (Années 1945-1985). Canton de Fribourg.

Conseil d'État de Neuchâtel (Hrsg.). Rapports du Conseil d'Etat au Grand Conseil sur sa gestion et l'exécution des lois (Années 1945-1985). Conseil d'État.

Historische Statistik der Schweiz (HSSO). (1996). Tab. Z.8b. Landwirtschaftliche Fachschulen nach Kantonen: Schülerbestand an den Jahres- und Winterschulen 1961-1986. Zugriff am 10.07.2019 unter hsso.ch/1996/z/8b.

Historische Statistik der Schweiz (HSSO). (2012). Tab. B.7. Wohnbevölkerung der Kantone nach Fünfjahresaltersklassen (annähernde Altersjahre) 1860-1990. Zugriff am 20.07.2019 unter hsso.ch/2012/b/7

Kanton Luzern (Hrsg.). Staatsverwaltungsbericht des Kantons Luzern (Ausgaben 1949-1986). Keller, [dann] Staatskanzlei.

Landwirtschaftliches Bildungs- und Beratungszentrum (LBBZ) Wilisau (Hrsg.). (1997). 75 Jahre Landwirtschaftsschule. 50 Jahre Bäuerliche-hauswirtschaftliche Fachschule. 35 Jahre Schulgutsbetrieb Burgrain. LBBZ.

Service de statistique de l'État de Fribourg (Hrsg.). (1990). Annuaire statistique du canton de Fribourg. Imprimerie Fragnières SA.

Statistisches Amt Basel-Stadt (25.10.2016). Sitzverteilung Grosser Rat des Kantons Basel-Stadt seit 1902. Zugriff am 14.09.2020 unter http://www.grosserrat.bs.ch/de/der-grosse-rat/geschichte/entwicklung-der-parteienlandschaft

Statistisches Amt Basel-Stadt (Hrsg.). Statistisches Jahrbuch des Kantons Basel-Stadt (Ausgaben 1949-1986). Statistisches Amt Basel-Stadt.

\section{Literatur}

Arnold, J. P. (2013). Die pfadabhängige Entwicklung im schweizerischen Bildungswesen: der Einfluss des Kulturkampfes auf das Zentralschweizer Bildungssystem, 1882 bis heute. Dissertation, Universität Zürich. https://doi.org/10.5167/uzh-164216

Barrelet, J.-M. (2002). La création d'une République. De la révolution de 1848 à nos jours (Histoire du canton de Neuchâtel, Tome 3). Alphil - Presses universitaires suisses.

Bays, F., Cottet, C., Philipona, A. \& Steinauer, J. (2016). Former des apprentis. L'enseignement dans le canton de Fribourg. Société d'histoire du canton de Fribourg, Service de la formation professionnelle SFP.

Becker, R. \& Schoch, J. (2018). Soziale Selektivität. Empfehlungen des Schweizerischen Wissenschaftsrates SWR (Politische Analyse 3/2018). SWR. https://www.swir.ch/images/stories/pdf/de/Politische_Analyse_SWR_3_2018_SozialeSelektivitaet_ WEB.pdf

Becker, R. \& Zangger, C. (2013). Die Bildungsexpansion in der Schweiz und ihre Folgen. Eine empirische Analyse des Wandels der Bildungsbeteiligung und -ungleichheiten mit den Daten der Schweizer Volkszählungen 1970, 1980, 1990 und 2000. Kölner Zeitschrift für Soziologie und Sozialpsychologie, 65(3), 423-449. https://doi.org/10.1007/s11577-013-0209-6

Becker, R. \& Zangger, C. (2016). Die Bildungsexpansion in der Schweiz - eine Reanalyse unter besonderer Berücksichtigung geschlechtsspezifischer Bildungschancen. Swiss Journal of Sociology, 42(3), 429-441.

Bonoli, L., Berger, J.-L. \& Lamamra, N. (Hrsg.). (2019). Enjeux de la formation professionnelle en Suisse. Le «modèle» suisse sous la loupe. Seismo.

Brühwiler, I., Criblez, L., Crotti, C., Helfenberger, M., Hofmann, M. \& Manz, K. (Hrsg.). (in Druck). Schweizer Bildungsgeschichte - Systementwicklung im 19. und 20. Jahrhundert (Historische Bildungsforschung, Band 5). Chronos.

Büchel, K. (2018). Duale Berufsbildung als Teil der «Bildungsexpansion». Der Kanton Luzern als Beispiel für die expansive Entwicklung der dualen Berufsbildung von 1954 bis 1980. Dissertation, Universität Zürich. Verfügbar unter https://doi.org/10.5167/ uzh-156966

Bühler, R. (2016). Eine neue Politik für eine neue Generation. Zum Wandel des Politikfelds Jugend in der Schweiz, 1960-1980. In L. Criblez, C. Rothen \& T. Ruoss, (Hrsg.), Staatlichkeit in der Schweiz. Regieren und verwalten vor der neoliberalen Wende (Historische Bildungsforschung, Band 2, S. 271-296). Chronos.

Criblez, L. \& Magnin, C. (2001). Editorial. Die Bildungsexpansion in der Schweiz der 1960er- und 1970er-Jahre. Schweizerische Zeitschrift für Bildungswissenschaften, 23(1), 5-12. https://doi.org/10.24452/sjer.23.1.4594

Criblez, L. (2001). Bildungsexpansion durch Systemdifferenzierung - am Beispiel der Sekundarstufe II in den 1960er- und 1970er-Jahren. Schweizerische Zeitschrift für Bildungswissenschaften, 23(1), 95-118. https://doi.org/10.24452/sjer.23.1.4598

Criblez, L. (2008). Bildungsraum Schweiz. Historische Entwicklung und aktuelle Herausforderungen. Haupt.

Criblez, L. (2015). Bildungsreformen und die Neukonstituierung der Schweizer Bildungsforschung in den 1960er- und 1970erJahren [Elektronische Version]. Pedagógiatörténeti Szemle, 1(4), 23-39. https://doi.org/10.22309/PTSZEMLE.2015.4.2

Criblez, L., Giudici, A., Hofstetter, R., Manz, K. \& Schneuwly, B. (Hrsg.). (in Druck). Die schulische Wissensordnung im Wandel. Schulfächer, Lehrpläne und Lehrmittel (Historische Bildungsforschung, Band 6). Chronos.

Degen, B. (2011). Von Pionier- zu Zusatzleistungen. Kantonale Sozialpolitik seit Mitte des 19. Jahrhunderts. In J. Mooser \& S. Wenger (Hrsg.), Armut und Fürsorge in Basel. Armutspolitik vom 13. Jahrhundert bis heute (S. 143-165). Christoph Merian.

Dorand, J.-P. (2017). La politique fribourgeoise au 20ème siècle. Presses polytechniques et universitaires romandes.

Ebel, M. (1981, mars). Femmes et formation professionnelle. Quelle réalité? Quel avenir? [Brochure]. Canton de Neuchâtel.

Felder, P. (2019). Für alle! Die Basler Volksschule seit ihren Anfängen. Schwabe.

Fischer, R. (2013). Schule und Bildung - der lange Weg zum Bildungszentrum. In K. Hürlimann \& J. Schmutz (Hrsg.), Der Kanton Luzern im 20. Jahrhundert (Band 1, S. 245-281). Chronos.

Gachet, D. (2016). La démocratisation de l'enseignement secondaire du degré inférieur à Fribourg. Analyse des débats politiques autour de la naissance du Cycle d'Orientation, 1950-1986. Mémoire de master non publié, Université de Fribourg.

Garufo, F. (2015). L'emploi du temps. L'industrie horlogère suisse et l'immigration (1930-1980). Antipodes.

Gonon, P. (2002). Arbeit, Beruf und Bildung. hep.

Green, N. L. (1990). L'histoire comparative et le champ des études migratoires [Version numérique]. Annales. Economies, sociétés, civilisations, 45(6), 1335-1350. 
Gugerli, D. \& Tanner, J. (2012). Wissen und Technologie. In P. Halbeisen, M. Müller und B. Veyrassat (Hrsg.), Wirtschaftsgeschichte der Schweiz im 20. Jahrhundert (S. 265-318). Schwabe.

Hadjar, A. \& Becker, R. (Hrsg.). (2006). Die Bildungsexpansion. Erwartete und unerwartete Folgen. Springer VS.

Häfliger, A. (2002). Der Luzerner Erziehungsrat 1798 - 1999. Eine schulhistorische Skizze. Luzerner Bildungsdepartement.

Helbig, M. \& Schneider, T. (2014). Auf der Suche nach dem katholischen Arbeitermädchen vom Lande. Religion und Bildungserfolg im regionalen, historischen und internationalen Vergleich. Springer VS. https://doi.org/10.1007/978-3-658-06282-8

Herren, M. (2008). Die nationale Hochschul- und Forschungspolitik in den 1960er- und 1970er Jahren. In: L. Criblez (Hrsg.), Bildungsraum Schweiz. Historische Entwicklung und aktuelle Herausforderungen (S. 219-250). Haupt.

Hofstetter, R. \& Schneuwly, B. (Hrsg.). (2011). Zur Geschichte der Erziehungswissenschaften in der Schweiz vom Ende des 19. bis zur Mitte des 20. Jahrhunderts. hep.

Hürlimann, G. (2013). Strukturen und Konjunkturen - ein Überblick. In K. Hürlimann \& J. Schmutz (Hrsg.), Der Kanton Luzern im 20. Jahrhundert (Band 1, S. 285-299). Chronos.

Imlig, F., Lehmann, L. \& Manz, K. (Hrsg.). (2018). Schule und Reform. Veränderungsabsichten, Wandel und Folgeprobleme (Educational Governance, Band 39). Springer VS.

Julien, E. (2005). Le comparatisme en histoire. Rappels historiographiques et approches méthodologiques [Version numérique]. Hypothèses, 8(1), 191-201. Consulté le 25 janvier 2021 dans https://www.cairn.info/revue-hypotheses-2005-1-page-191.htm

Kreis, G. (2000). Goldene Jahre mit irritierenden Erfahrungen. In G. Kreis \& B. von Wartburg (Hrsg.), Basel - Geschichte einer städtischen Gesellschaft (S. 268-312). Christoph Merian.

Lorenzetti, L. (2012). Demographie und Wirtschaftsentwicklung. In P. Halbeisen, M. Müller und B. Veyrassat (Hrsg.), Wirtschaftsgeschichte der Schweiz im 20. Jahrhundert (S. 223-264). Schwabe.

Magnin, C. \& Muller, C. A. (Hrsg.). (2012). Enseignement secondaire, formation humaniste et société XVIe-XXIe siècle (Acte du colloque international et pluridisciplinaire tenu à l'occasion du 450e anniversaire de la fondation du Collège de Genève par Calvin). Slatkine.

Mantovani Vögeli, L. (1994). Fremdbestimmt zur Eigenständigkeit. Mädchenbildung gestern und heute. Rüegger.

Mooser, J. (2000). Konflikt und Integration - Wirtschaft, Gesellschaft und Politik in der «Wohlfahrtsstadt». In G. Kreis \& B. von Wartburg (Hrsg.), Basel-Geschichte einer städtischen Gesellschaft (S. 226-263). Christoph Merian.

Osterwalder, F. (2018). Einsiedeln - Bern - Zürich über Genf. Die lange Dauer von Bildungsreformen. In F. Imlig, L. Lehmann \& K. Manz (Hrsg.), Schule und Reform. Veränderungsabsichten, Wandel und Folgeprobleme (Educational Governance, Band 39, S. 77-88). Springer.

Pätzold, G. (2008). Übergang Schule - Berufsausbildung. In W. Helsper \& J. Böhme (Hrsg.), Handbuch der Schulforschung (2., durchg. u. erw. Aufl., S. 593-610). VS.

Peisert, H. (1967). Soziale Lage und Bildungschancen in Deutschland. Piper.

Pfenninger, P. (1998). Zweihundert Jahre Luzerner Volksschule 1798-1998 (Begleitheft zur Sonderausstellung «Von der Schiefertafel zum Computer. Zweihundert Jahre Schule für das Volk» im Historischen Museum Luzern, 27. Mai bis 8. November 1998). Historisches Museum.

Piérart, A. (2005). De la tradition agricole à la stratégie industrielle. La politique de développement économique du canton de Fribourg durant les Trente Glorieuses (1945-1971). Mémoire de licence non publié, Université de Fribourg.

Praz, A.-F. (2006). Filles et garçons à l'école vers 1900. Discours et pratiques dans les cantons de Vaud et Fribourg. In A. Dafflon Novelle (Hrsg.), Filles-garçons, Socialisation différenciée? (S. 103-126). Presses Universitaires.

Python, F. (2002). Histoire de Fribourg. Ancrages traditionnels et renouveaux (XIXe-XXe siècle) (Tome 3). Livreo-Alphil.

Rieger, A. (2001). Bildungsexpansion und ungleiche Bildungspartizipation am Beispiel der Mittelschulen im Kanton Zürich, 1830 bis 1980. Schweizerische Zeitschrift für Bildungswissenschaften, 23(1), 41-72.

Ruoss, T. (2018). Zahlen, Zählen und Erzählen in der Bildungspolitik. Lokale Statistik, Politische Praxis und die Entwicklung städtischer Schulen zwischen 1890 und 1930 (Historische Bildungsforschung, Band 4). Chronos.

Schoepfer, A. (1982). L'économie du canton de Neuchâtel entre 1970 et 1980. Éléments de l'évolution et de la répartition de la structure industrielle démontrés sur la base des études déjà publiées et illustrées à l'exemple des industries de la métallurgie et des machines/ appareils. Mémoire de licence non publié, Université de Neuchâtel.

Société d'histoire et d'archéologie du canton de Neuchâtel (1993). Histoire du pays de Neuchâtel. De 1815 à nos jours (Tome 3). Gilles Attinger.

Stirnimann, C. (2000). Die Öffnung des Bildungssystems. Vom Schulgesetz von 1880 zur Bildungsexpansion der 1960er-Jahre. In G. Kreis \& B. von Wartburg (Hrsg.), Basel-Geschichte einer städtischen Gesellschaft (S. 384-394). Christoph Merian.

Tabin, J.-P. (1989). Formation professionnelle en Suisse. Histoire et actualité. Réalités sociales.

Tanner, J. (2015). Geschichte der Schweiz im 20. Jahrhundert. C.H. Beck.

Vuagniaux, R. (2011). Des biais de genre dans les indicateurs du travail. In A.-F. Praz \& S. Burgund (Hrsg.), Genre et bien-être. Questionner les inégalités (S. 44-58). Seismo.

Zangger, C., \& Becker, R. (2016). Die Bildungsexpansion in der Schweiz - eine Reanalyse unter besonderer Berücksichtigung geschlechtsspezifischer Bildungschancen. Swiss Journal of Sociology, 42(3), 429-441. https://doi.org/10.1515/sjs-2016-0019

Schlagworte: Bildungsteilhabe; Bildungsexpansion; Sekundarstufe II; Berufsausbildung; Bildungsgeschichte 


\section{L'influence des changements économiques et sociaux sur la participation des jeunes à l'éducation à Bâle-Ville, Fribourg, Lucerne et Neuchâtel 1950-1985}

\section{Résumé}

Cet article traite la participation des jeunes de 15 à 19 ans à l'éducation pendant l'expansion de l'enseignement. Les auteurs prennent en compte la structure fédérale de l'éducation en comparant les quatre cantons de Bâle-Ville, Fribourg, Lucerne et Neuchâtel. Ainsi, elles retracent l'évolution de la répartition quantitative-statistique des jeunes dans l'enseignement post-obligatoire entre 1950 et 1980 et l'expliquent sur la base de facteurs économiques, sociopolitiques et éducatifs. Il ressort que l'expansion de l'éducation a été un développement très différencié qui dépendait des circonstances cantonales.

Mots clés : Participation à l'éducation ; expansion de l'enseignement ; degré secondaire II ; formation professionnelle; histoire de l'éducation

\section{Impatto del cambiamento economico e sociale sulla partecipazione scolastica dei giovani a Basilea Città, Friburgo, Lucerna e Neuchâtel 1950-1985}

\section{Riassunto}

Questo articolo si occupa della partecipazione scolastica dei ragazzi dai 15 ai 19 anni durante la fase di ampliamento delle opportunità educative. Nello studio le autrici prendono in considerazione la struttura federale del sistema dell'istruzione confrontando i quattro cantoni di Basilea Città, Friburgo, Lucerna e Neuchâtel. Rispetto a tali cantoni, tracciano l'evoluzione della distribuzione quantitativo-statistica dei giovani nell'istruzione postobbligatoria tra il 1950 e il 1980 e la spieghiamo sulla base di fattori economici, sociopolitico e di politica educativa. Sulla base dei dati raccolti s può dimostrare che l'ampliamento delle opportunità educative fu uno processo altamente differenziato che dipese in modo decisivo dalle circostanze cantonali.

Parole chiave: Partecipazione scolastica; ampliamento delle opportunità educative; livello secondario II; formazione professionale; storia dell'educazione

\section{Impact of economic and social change on the educational participation of young people in Basel-City, Fribourg, Lucerne and Neuchâtel 1950-1985}

Summary

This article deals with the educational participation of 15- to 19-year-olds during the period of educational expansion. The authors consider the federal structure of the education system by comparing the four cantons of Basel-Stadt, Fribourg, Lucerne and Neuchâtel. In doing so, they trace the development of the quantitativestatistical distribution of young people in post-compulsory education between 1950 and 1980 and explain it based on economic, social and educational factors. It can be shown that the expansion of education was a highly differentiated development that depended decisively on the cantonal circumstances.

Keywords: Educational participation; educational expansion; upper secondary level; vocational training; history of education 
Miriam Baumeister, M.A. in Geschichte und Kulturanthropologie, ist Doktorandin im Rahmen des NFP 76 an der Pädagogischen Hochschule Luzern und der Basel Graduate School of History der Universität Basel. Institut Geschichtsdidaktik und Erinnerungskulturen (IGE), PH Luzern, Frohburgstrasse 3, CH-6002 Luzern E-Mail: miriam.baumeister@phlu.ch,miriam.baumeister@unibas.ch

Valérie Bürgy, M.A. in Kulturwissenschaften mit Schwerpunkt Geschichte, ist freischaffende Kulturwissenschaftlerin sowie Studentin spezialisierter Master in Geschichtsdidaktik und öffentliche Geschichtsvermittlung an der Pädagogischen Hochschule Luzern und Universität Freiburg.

Institut Geschichtsdidaktik und Erinnerungskulturen (IGE), PH Luzern, Frohburgstrasse 3, CH-6002 Luzern E-Mail: valerie.buergy@stud.phlu.ch

Aurore Müller, M.A. in Zeitgeschichte, ist wissenschaftliche Mitarbeiterin und Doktorandin im Rahmen des NFP 76 an der Universität Freiburg.

Département Histoire contemporaine, Université de Fribourg, Av. de l'Europe 20, CH-1700 Fribourg

E-Mail: aurore.mueller@unifr.ch 